\title{
Input protection for the laboratory computer
}

\author{
STANLEY A. HERTEL and STEPHEN E. EDGELL \\ University of Louisville, Louisville, Kentucky
}

\begin{abstract}
Circuit details are given for building protective interfaces between laboratory instruments and a computer's analog and digital inputs. The protective interfaces will not only protect the computer and its input interfaces from a misconnection, but they will themselves withstand misconnection with no damage.
\end{abstract}

Although the growing utility of inexpensive computers has led to their extensive use in research laboratory automation, the electrical protection of signal input ports to computer systems is frequently overlooked by researchers. Dedicated systems, properly installed and operating permanently in fixed configurations, often do not require input protection. Many laboratories, however, cannot afford separate, dedicated processors for each ongoing experiment. In some laboratories, a single computer system is shared among many experiments and occasionally among several experimenters. As various work is initiated and concluded, digital and analog signal inputs are routinely changed, frequently by users with no appreciation for interface hardware limitations. Because a wide variety of equipment is used in psychology laboratories, there is little signal standardization in the instrumentation. Experience has shown that even careful workers sometimes make erroneous connections. Mistaken connection of excessive input voltages to electrically fragile computer interfaces can destroy them, and possibly even propagate failure into the host computer itself. Furthermore, laboratory equipment can fail, sometimes presenting excessive voltages to downstream computer interfaces. Regardless of the failure mode, the resulting downtime can be expensive, both in terms of cost of repair and lost laboratory time. It is prudent to implement robust analog and digital input circuits that will handle overloads with no adverse consequences either to themselves or to interfaces and computers downstream. Using this approach, otherwise catastrophic misconnections simply result in temporarily nonfunctional, but undamaged, inputs.

It is unlikely that students of electronic design will be intrigued by the following circuits. However, psychologists and other researchers who use computers to instrument their laboratories should find them to be a useful investment. Consistent with adequate performance, our primary design criteria in this project were circuit simplicity and ease of construction. In our laboratories this

The authors wish to thank William I. Latta, Jr., for his excellent construction and testing work on this project. Communications regarding this article should be sent to either author at Department of Psychology, University of Louisville, Louisville, KY 40292. approach has enhanced long-term reliability, minimized repairs, and eliminated the accidental destruction of equipment.

\section{INPUT PROTECTION AND ISOLATION}

Although both are desirable, protection and isolation are separate electrical characteristics. A protected input is one that can, within reasonable limits, tolerate connection to a signal in excess of normal specifications and then return to normal operation once the offending signal is removed. A protected input thus performs a buffering action when presented with an excessive input, inhibiting the propagation of such a potentially damaging signal to downstream computer electronics. The protection circuits described later merely saturate in the presence of excessive signals, rendering them temporarily inoperative. Their saturated outputs under this condition are harmless to downstream connected computer hardware.

An isolated input, on the other hand, may or may not also be protected and is characterized by the absence of ohmic continuity in signal transmission and ground paths. An isolated stage breaks input-signal ohmic continuity at the isolation barrier, yet allows accurate signal transmission to its output. Such isolation provides electrical separation of an input fault from downstream electronics. Note that, if unprotected, this stage may itself become damaged by an excessive input, but the desired isolation prevents failure propagation further into the computer system.

Ideally, input protection and isolation circuits should tolerate, without damage, erroneous connections to $120 \mathrm{~V}$ ac. An ac power line presents the largest voltage source found in most psychology laboratories. Furthermore, such circuits should not significantly bias the experimental procedure. Speed and accuracy of signal propagation through these circuits must be acceptable with respect to experimental tolerances. This paper describes construction details for protecting and isolating digital inputs that offer such performance. A similarly ideal arrangement for ana$\log$ inputs, unfortunately, is more difficult. To minimize circuit complexity and cost, therefore, the analog inputs described are protected but do not provide isolation.

There have been several articles in this journal that have advocated the use of optical isolators to isolate the 
computer's digital inputs, and in some papers the outputs, from the laboratory environment (Algarabel, Sanmartin, \& Ahuir, 1989; Campbell \& Forest, 1984; Gordon, Foree, \& Eckerman, 1983; Grisham \& Frei, 1977; Palya \& Doyle, 1978; Rayfield \& Carney, 1981; Rosellini, Carlson, Dina, Falcone, \& Shapiro, 1983; Scoles, 1990; Solomon, Weisz, Clark, Hall, \& Babcock, 1983; Stoddard \& Loftus, 1988). However, all of the circuits presented in these articles would suffer damage if $120 \mathrm{~V}$ ac were applied to them. They would protect the downstream digital interfaces and the computer, but considerable time could be lost repairing the isolation circuits. The circuits presented in the present paper avoid that problem. Furthermore, we have not found an article in this journal on protecting an analog input.

\section{COMMERCIALLY AVAILABLE HARDWARE}

Modular functional blocks that could simplify computer input protection or isolation are available from several commercial equipment manufacturers. Such input modules generally process one analog or digital signal each, with multichannel operation facilitated by the stacking of individual modules in groups on a universal motherboard. Input modules are attached as required to the appropriate motherboard, which distributes power throughout the system and provides convenient signal connections. Such an arrangement, then, could conveniently be connected between various laboratory instruments and the laboratory's computer system. On initial appearance, this seems an ideal solution to computer input protection and isolation, except for two problems: (1) Although available analog input modules offer isolation and are self-protecting, they are expensive, costing approximately $\$ 2,500$ for a 16 channel system; and (2) digital modules, although offering isolation, have not been found conveniently available with protected inputs. In-house design of the required electronics was therefore done. ${ }^{1}$

\section{DIGITAL INPUT PROTECTION AND ISOLATION}

A common computer interface in the psychology laboratory is one that inputs digital information. These are used in collecting subjects' responses via key or push-button switches or in the monitoring of various instruments that output data as binary information. In the circuits to follow, nominal inputs from the laboratory and outputs to the computer digital interface are 0 to $+5 \mathrm{~V}$ dc, voltage levels compatible with most commercially available TTL and 5-V CMOS interfaces. Digital input interfaces available for computers such as the IBM PC, the Macintosh, and DEC computers typically use this voltage range. Propagation time is $100 \mu \mathrm{sec}$ or less, well within psychology laboratory standards. Given proper construction, the circuits described will withstand misconnection or other inadvertent application of voltages up to $120 \mathrm{~V}$ ac for an indefinite time with no damage to themselves or to downstream interfaces and computers. One need only remove the offending voltage by correcting the misconnection to restore full functioning of the laboratory computer system. They further provide isolation that will prevent damage to downstream interfaces and computers for even larger voltages. While the illustrated design provides for eight input bits, the circuit of Figure 3 can be reproduced as necessary to provide additional inputs. To facilitate hardware minimization, these circuits logically invert input signals, this usually being of little software consequence. However, referring to Figure 2 , inverters could be applied at Circuit B output points " $G$ " and " $H$ " by those desiring positive true assertion.

\section{Digital Input Circuit}

Circuit A and Circuit B are shown in Figures 1 and 2, respectively. Incorporation of these into a completed circuit is shown in Figure 3. Layout of components is not critical, and, for convenience, the circuits may be built from left to right as drawn. Glass-epoxy, perforated circuit board, and "push-through" solder terminals are suggested for component mounting and point-to-point wiring. The use of circuit board with perforations on 0.1 -in. centers will facilitate mounting of sockets for all integrated circuits.

Figure 3 illustrates the arrangement of eight input protection channels into an eight-bit system. Input connectors are chassis isolated BNC style, Amphenol 31-10, or equivalent. To achieve the desired isolation, it is necessary that connectors that are isolated from the chassis be used. Miniature coaxial cable, Belden 8216, is used between these and the Circuit $\mathrm{A}$ inputs. Connections to the laboratory computer are machine specific and are left to the builder's discretion. Belden 8216 cables of length as short as practical are recommended.

\section{Digital Input Theory of Operation}

A representative channel of input protection consists of an input clamp circuit, a buffer, and an optoisolator. The

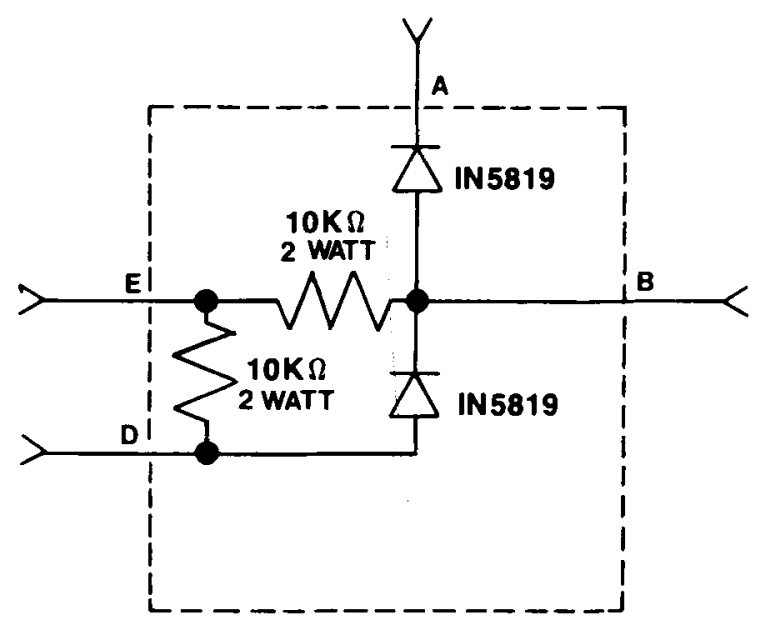

Figure 1. Circuit A, input clamp circuit for a digital input channel. All resistors are $2-\mathrm{W}$, carbon-composition types, $10 \%$ tolerance. 


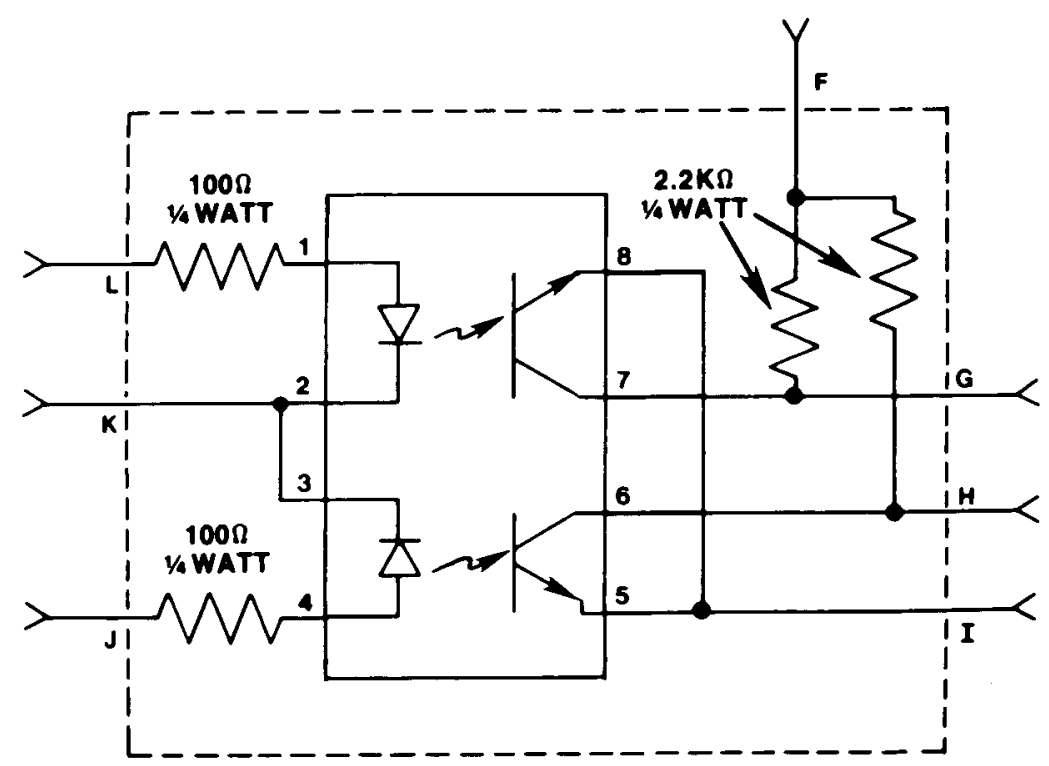

Figure 2. Circuit B, isolation circuits for two digital input channels. The dual-channel optical isolator is a General Instrument MCT6. All resistors are 1/4W, carbon-composition types, $10 \%$ tolerance.

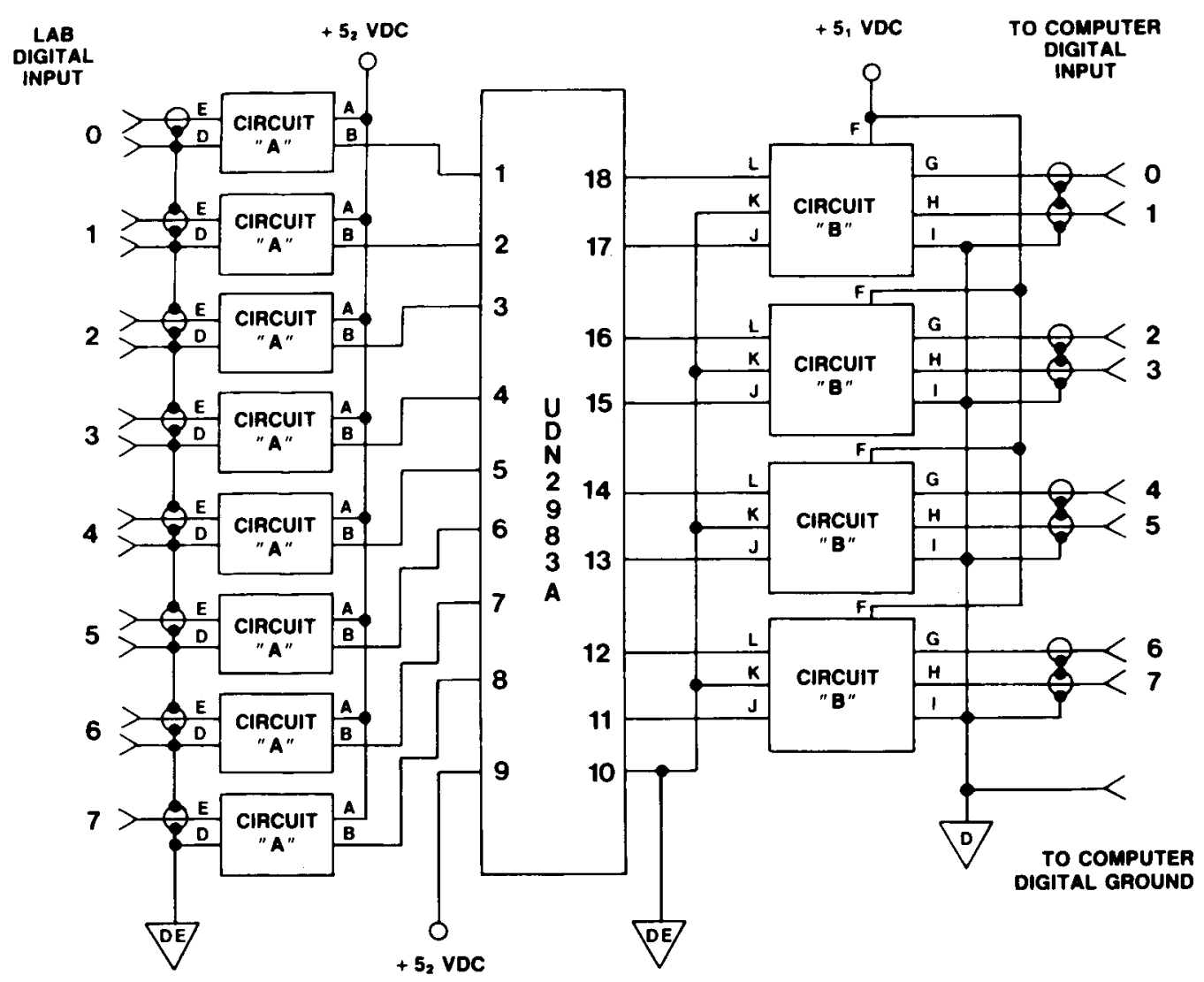

Figure 3. Eight-bit digital input protection/isolation system. 
input clamp utilizes two schottky diodes to limit voltage excursions at point " $B$ '" of Circuit $A$, if an input applied between points " $E$ "' and ' $D$ " exceeds the nominal 0 to $+5 \mathrm{~V} \mathrm{dc}$ range. Excess voltage is dropped across the $10-k \Omega$ resistor in series between points " $E$ "' and " $B$." A second $10-\mathrm{k} \Omega$ resistor terminates the input, should no laboratory device be connected. Note that both $10-\mathrm{k} \Omega$ resistors must be rated to dissipate at least 2 W RMS.

Point " $B$ " of the input clamp drives a noninverting buffer contained in a Sprague UDN2983A source driver integrated circuit. Eight such buffer stages are provided by a single UDN2983A; this integration facilitates ease of construction and maintenance. (The Sprague UDN2981A may be substituted for the UDN2983A in this application. A Mitsubishi Electronic Corporation M54563P can also be substituted. It should be noted that the authors have not tested these substitutions, but Sprague indicates that the UDN2981A and the M54563P are equivalent.)

An optically isolated transistor, one of two contained by an MCT6 optoisolator, is driven by each buffer's output. This stage, shown as Circuit B, along with the power supply arrangement discussed later, mechanizes an isolated coupling to the laboratory computer interface. A 2.2-k $\Omega$ pull-up resistor to $+5 \mathrm{~V} \mathrm{dc}$ is used at each isolator's output, thus providing the desired voltage levels.

The oscilloscope photographs of Figures 4 and 5 illustrate representative propagation delays introduced by the circuits of Figure 3. In each case, an input applied between points " $E$ " and " $D$ " of Circuit $A$ is shown as the top trace. Similarly, in each case, the bottom trace represents an output measured between points " $G$ " and " $I$ " of Circuit B. (Recall that the circuits are inverting.) Characteristics for positive- and negative-going inputs are shown in Figures 4 and 5, respectively. A time base of $1 \mu \mathrm{sec}$ per major division is represented in Figure 4, and $10 \mu \mathrm{sec}$ per major division is illustrated in Figure 5. As can be seen in the figures, the time delays are well within those tolerable in psychology laboratory settings. Nominal switching of the digital input circuit occurs at approximately $0.7 \mathrm{~V}$ dc. Figures 4 and 5 illustrate the propagation delays caused by the buffer and optical isolator circuits but do not clearly illustrate the switching point.

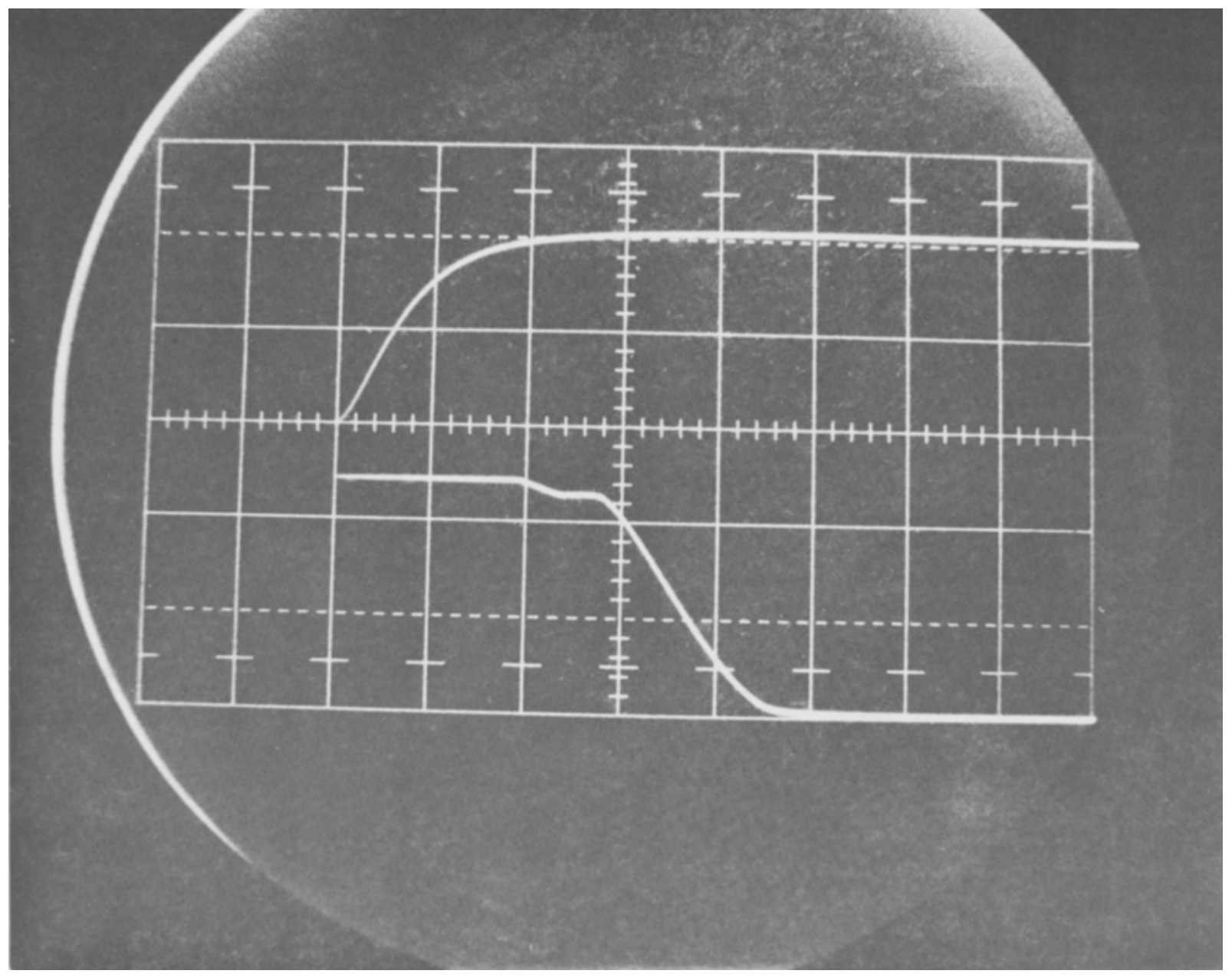

Figure 4. Digital input protection/isolation channel transient response for a positive-going input. Top and bottom traces are channel input and output, respectively. Oscilloscope calibrations are: vertical, 2 V/division and horizontal, $1 \mu$ sec/division. 


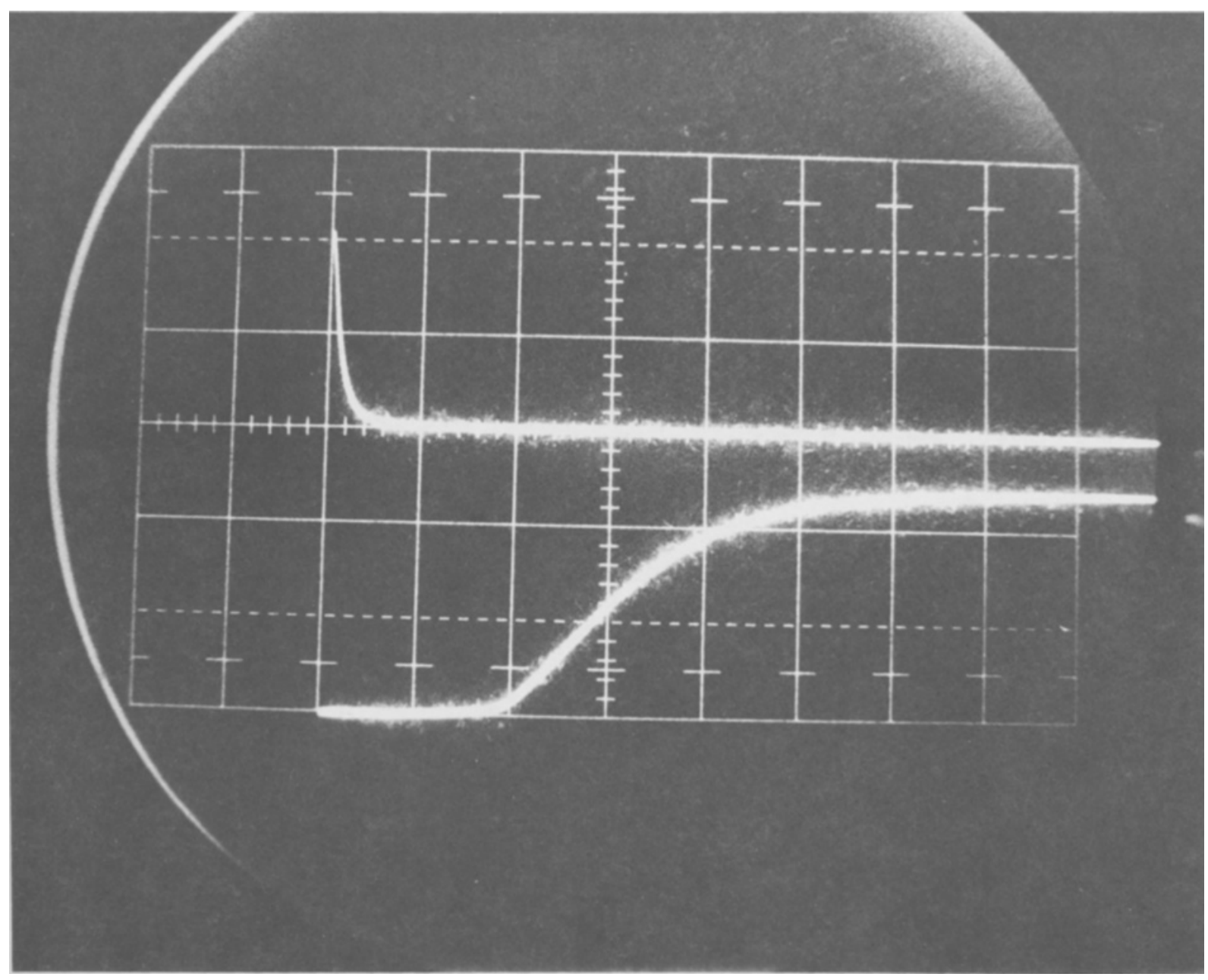

Figure 5. Digital input protection/isolation channel transient response for a negative-going input. Top and bottom traces are channel input and output, respectively. Oscilloscope calibrations are: vertical, 2 V/division and horizontal, $10 \mu \mathrm{sec} / \mathrm{division.}$

\section{Digital Input Power Supplies}

To enhance electrical isolation of the laboratory computer from connected digital instrumentation, two power supplies are used. This is desirable in addition to optical isolation of input channels as it reduces the possibility of damaging voltages migrating through a single, common supply. Notice that 5-V dc Power Supply No. 1 (" $+5, V$ dc") is used on the computer side of Circuit B, while 5-V dc Power Supply No. 2 (" $+5_{2} \mathrm{~V} \mathrm{dc}$ ") is used on the laboratory side. Circuit grounds for these supplies are separate, being annotated " $D$ " (digital) for Supply No. 1 and "DE" (digital experimenter) for Supply No. 2.

It is recommended that commercially available enclosed, transformer-operated power supplies be used. A line and load regulation of $1 \%$ is desirable, along with overcurrent and overvoltage protection. The supplies should each have a continuous duty rating of $1 \mathrm{~A}$ for an eightchannel protection system and should be fused per manufacturer's recommendations.

\section{ANALOG INPUT PROTECTION}

Analog and digital inputs differ in that the former may assume any value through a valid input range rather than just one of two values representing a logical true or false. Hence, in protecting a laboratory computer's analog inputs, not only are circuit delays of concern, but also errors in circuit gain and offset (which include deviations from linearity). Gain is defined as the protection circuit's amplification factor, the ratio of output to input within its linear operating range. Offset describes the circuit's dc output when zero input exists. Protection circuit delay, gain, and offset errors combine with those of the computer's analogto-digital converter to determine the performance of the entire A/D system. Again, as with the digital protection circuits, these circuits, if properly constructed, will withstand for an indefinite length of time misconnection to voltages up to $120 \mathrm{~V}$ ac with no damage either to the circuits or to downstream interfaces and computers. 


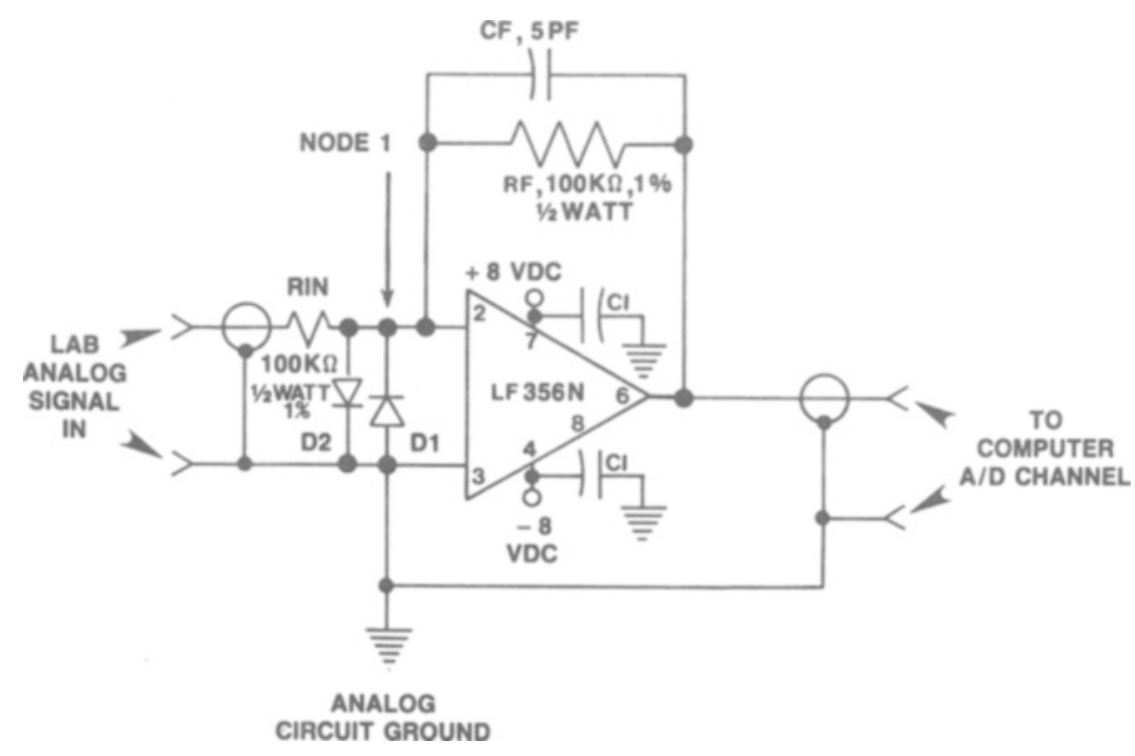

Figure 6. Analog input protection channel. All resistors are 1/2-W, metal-film type, $100 \mathrm{k} \Omega 1 \%$ tolerance. Diodes D1 and D2 are type 1N4148. Capacitor CF is a silver-mica type, $5 \mathrm{pF}, 5 \%$ tolerance. Capacitors $\mathrm{C} 1$ are disk-ceramic type, $0.1 \mu \mathrm{F}, 20 \%$ tolerance. All capacitors are rated at 50 WV dc.

\section{Analog Input Circuit}

Circuit $\mathbf{C}$, a representative analog input protection amplifier, is shown in Figure 6. One such stage is required for each channel in the computer's A/D conversion system. Note that the analog circuit ground is separate from either of the digital input grounds discussed earlier.

Glass-epoxy board with terminals, point-to-point wiring, and sockets for all integrated circuits are again recommended in construction. Multiple protection amplifiers, one for each computer analog input, may be constructed on one board. However, care should be taken to place each channel's components as close as possible to their respective LF356N. Each channel's inputs and outputs should be located on opposite ends of the circuit board, thus avoiding a $U$-shaped construction that places inputs closely adjacent to outputs. Furthermore, as much as possible, each channel's wiring should be well separated from its neighbor's. A long and narrow circuit board with individual channels spaced into a single row is suggested.

Input connectors are chassis-isolated BNC style, Amphenol 31-10, or equivalent, with Belden 8216 cable used between these and all Circuit $C$ inputs. To achieve the desired isolation, it is necessary that connectors isolated from the chassis be used. Connections to the laboratory computer are again left to the builder's discretion. Belden 8216 cables of length as short as practical are recommended.

\section{Analog Input Theory of Operation}

Our laboratory computer's analog input range is specified as a bipolar range of -5 to $+5 \mathrm{~V}$ dc. Most commercially available analog to digital converters are setable to operate within this range. Circuit $\mathrm{C}$ has thus been mechanized with an identical linear range. A National
Semiconductor LF356N is connected as an inverting stage with a nominal closed loop gain of -1 . To enhance circuit simplicity, no adjustment of amplifier gain or offset is provided. Operating software can conveniently compensate for small gain and offset errors as well as the amplifier's inverting characteristic.

Diodes D1 and D2, along with resistor RIN, implement the desired input protection. During normal operation, feedback characteristics of the amplifier hold Node 1 at ground potential. Should excessive signal be applied as input, however, either diode D1 or D2 becomes forward biased, limiting Node 1 to a voltage harmless to the LF356N. Diode D1 becomes active for inputs less than approximately $-6 \mathrm{~V}$ peak, while $\mathrm{D} 2$ activates for inputs greater than about $+6 \mathrm{~V}$. The difference in voltage thus developed between Node 1 and the input is dropped across resistor RIN. Under these overdrive conditions, protection amplifier power supplies of +8 and $-8 \mathrm{~V}$ dc limit the LF356N saturated output at values harmless to downstream computer analog inputs.

One-percent-tolerance resistors throughout the circuit have produced adequate performance for our needs. Neglecting offset, estimated worst-case dc gain error with these components is approximately $\pm 2 \%$ of nominal output. If necessary, this performance could be improved substantially by the use of $0.1 \%$-tolerance resistors. Of 17 protection amplifiers constructed thus far, maximum offset measured has been $+13 \mathrm{mV}$ dc. Amplitude frequency response at $3.5 \mathrm{~V}$ RMS sinusoidal input, within our measurement capability, is flat from dc to $20 \mathrm{kHz}$. The amplifier's measured phase shift remains constant at $-180^{\circ}$ (the inverting characteristic) from dc through $6 \mathrm{kHz}$. Higher input frequencies gradually produce ad- 


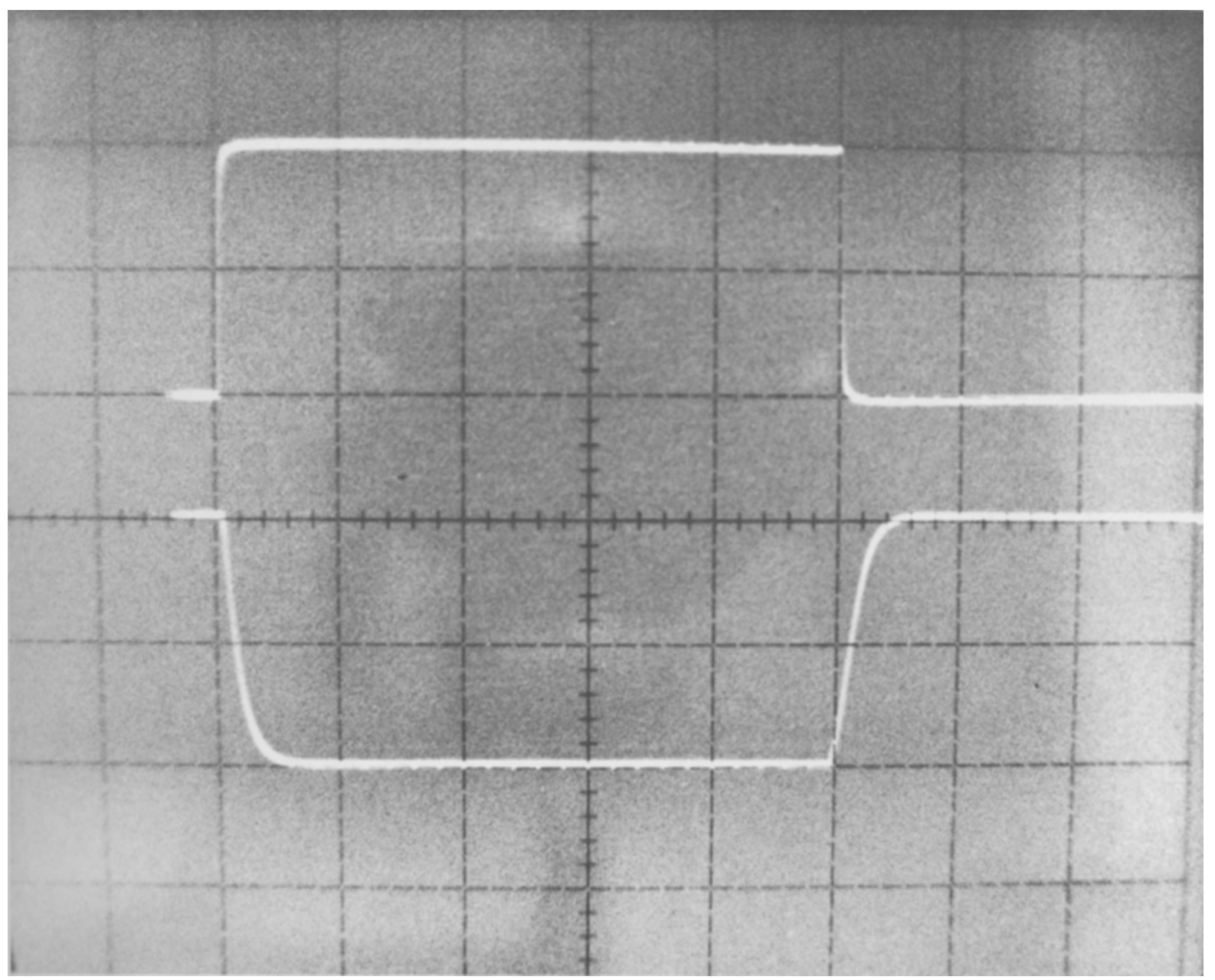

Figure 7. Analog input protection channel transient response. Top and bottom traces are channel input and output, respectively. Oscilloscope calibrations are: vertical, $2 \mathrm{~V} /$ division and horizontal, $2 \mu \mathrm{sec} / \mathrm{division}$.

ditional lag until at $20 \mathrm{kHz}$, the maximum frequency of interest in our laboratories, an additional phase lag of $5^{\circ}$ was measured. These operating characteristics are more than adequate for most psychology laboratory work.

Amplifier transient response is illustrated in Figure 7. Both input and output signals, the upper and lower traces, respectively, are referenced to analog circuit ground. As shown, the input is a ground referenced pulse of amplitude $+4 \mathrm{~V} \mathrm{dc}$ and length $10 \mu \mathrm{sec}$, with rise and fall times of $0.1 \mu \mathrm{sec}$. The resulting amplifier response is a pulse of similar length, having an amplitude of $-4 \mathrm{~V}$ dc (the amplifier's inverting characteristic) with rise and fall times of approximately $1 \mu \mathrm{sec}$. No amplifier overshoot is evident on the output trace. This transient performance is ample for the measurement of nerve action and early photoreceptor potentials, among the fastest signals of interest in psychology laboratories.

Protection amplifier input resistance, determined by RIN, is $100 \mathrm{k} \Omega$. We have found this to be sufficiently high for our needs. For laboratory instruments having output source resistances of $\mathbf{l} \mathbf{k} \Omega$ or less, error due to the loading effect of this input resistance will be less than $1 \%$.

\section{Analog Input Power Supplies}

It is recommended that commercially available enclosed, transformer-operated supplies be used as +8 and $-8 \mathrm{~V} \mathrm{dc}$ sources for the protection amplifiers. Note that these supplies are separate from the digital input circuit power sources previously discussed. Line and load regulation of at least $1 \%$ is desirable, along with overcurrent and overvoltage protection. Properly fused supplies each rated for continuous duty at $250 \mathrm{~mA}$ will be adequate for a 16-channel protection system.

\section{REFERENCES}

Algarabel, S., Sanmartin, J., \& AhUir, F. (1989). A voice-activated key for the Apple Macintosh computer. Behavior Research Methods, Instruments, \& Computers, 21, 67-72.

CAmpbell, D. J., \& Forest, J. (1984). The KIM-1 microcomputer as 
an eight-channel data logger. Behavior Research Methods, Instruments, \& Computers, 16, 315-319.

Gordon, W. A., Foree, D., \& EcKerman, D. A. (1983). Using an Apple II microcomputer for real-time control in a behavioral laboratory. Behavior Research Methods \& Instrumentation, 15, 158-166.

Grisham, M. G., FreI, L. J. (1977). An optically isolated digital interface for the SKED system. Behavior Research Methods \& Instrumentation, 9, 215-218.

Palya, W. L., \& Doyle, A. F. (1978). A relay driver and contact closure sensing interface for an LSI-11 DRV11 parallel I/O board. Behavior Research Methods \& Instrumentation, 10, 485-487.

RAYFIELD, F., CARNEY, J. (1981). Controlling behavior experiments with BASIC on 6502-based microcomputers. Behavior Research Methods \& Instrumentation, 13, 735-740.

Rosellini, R. A., Carlson, J., Dina, F., Falcone, R., \& Shapiro, N. R. (1983). A large-scale expandable I/O port interface for the TRS-80 microcomputer. Behavior Research Methods \& Instrumentation, 15, 45-48.

Scoles, M. T. (1990). The silicon-controlled rectifier as an input latch for digital laboratory computer interfaces. Behavior Research Methods, Instruments, \& Computers, 22, 302-304.
Solomon, P. R., Weisz, D. J., Clark, G. A., Hall, J., \& Babcock, B. A. (1983). A microprocessor control system and solid state interface for controlling electrophysiological studies of conditioning. $\mathrm{Be}$ havior Research Methods \& Instrumentation, 15, 57-65.

Stoddard, P. K., \& LofTUS, G. R. (1988). An IBM XT-compatible, computer-based, slide-projector laboratory. Behavior Research Methods, Instruments, \& Computers, 20, 541-551.

\section{NOTE}

1. For the circuits described in this paper, the cost of all components, including perforated board, push pins for the board, and all sockets, is $\$ 42$ for 8 channels (bits) of digital input and $\$ 41$ for 8 channels (bits) of analog output. This does not include the cost of wire, solder, connectors, cabling, and power supplies, which also would be required for any off-the-shelf commercial module.

(Manuscript received September 7, 1990; revision accepted for publication January 16, 1991.) 